\title{
Avaliação de guidelines para atenção pós-parto na Atenção Primária: revisão sistemática
}

\section{Guidelines evaluation for postpartum attention in Primary Care: systematic review}

\author{
Tatiane Baratieri' (D), Larissa Gramázio Soares² (D), Sonia Natal ${ }^{3}$ (D), Josimari Telino de Lacerda ${ }^{3}$ (D) \\ 'Departamento de Enfermagem, Universidade Estadual do Centro-Oeste (UNICENTRO) - Guarapuava (PR), Brasil. \\ 2 Programa de Pós-Graduação em Enfermagem, Universidade Federal do Paraná (UFPR) - Curitiba (PR), Brasil. \\ ${ }^{3}$ Departamento de Saúde Pública, Universidade Federal de Santa Catarina (UFSC) - Florianópolis (SC), Brasil.
}

Como citar: Baratieri T, Soares LG, Natal S, Lacerda JT. Avaliação de guidelines para atenção pós-parto na Atenção Primária: revisão sistemática. Cad Saúde Colet, 2021;29(4):616-623. https://doi.org/10.1590/1414-462X202129040051

\section{Resumo}

Introdução: O cuidado após o parto, denominado de pós-natal, pós-parto ou puerpério, é um importante período para desenvolvimento de estratégias de atenção à saúde das mulheres para redução da morbimortalidade. Com o aumento de publicações de diretrizes clínicas, há preocupações quanto à qualidade delas. Objetivo: Avaliar a qualidade de guidelines sobre a atenção às mulheres no pós-parto na Atenção Primária à Saúde. Método: Revisão sistemática de guidelines publicados entre 2008 e 2017, nas bases de dados Lilacs e PubMed, e página da internet do National Guideline Clearinghouse, National Institute for Health and Care Excellence e Google ${ }^{\circledast}$. A ferramenta Appraisal of Guidelines for Research and Evaluation /l foi utilizada para avaliação da qualidade dos guidelines nos domínios: escopo e finalidade; envolvimento das partes interessadas (stakeholders); rigor do desenvolvimento; clareza e apresentação; aplicabilidade; e independência editorial. Resultados: Foram analisados dez guidelines que apresentaram escore médio geral de avaliação de 77\%, com melhor avaliação para "escopo e finalidade" (92\%) e pior para "aplicabilidade" (57\%). Conclusão: Os guidelines apresentaram boa qualidade geral com necessidade de melhorar sua elaboração no domínio aplicabilidade.

Palavras-chave: período pós-parto; Atenção Primária à Saúde; guia de prática clínica.

\begin{abstract}
Background: Postpartum care, known as postnatal care, postpartum or puerperium, is an important period for the development of health care strategies for women aiming to reduce morbidity and mortality. With the increase of publications of clinical guidelines, there are concerns about their quality. Objective: To evaluate guidelines quality on women postpartum care in Primary Health Care. Method: Systematic review of guidelines published between 2008 and 2017, in Lilacs and PubMed databases, and the National Guideline Clearing House, National Institute for Health and Care Excellence and Google ${ }^{\circledR}$. Appraisal of Guidelines for Research and Evaluation II tool was used to assess the quality of the guidelines as to: scope and purpose; stakeholders involvement; accuracy of development; transparency and presentation; applicability; and editorial independence. Results: Ten 10 guidelines that presented a general average score of $77 \%$, with a better evaluation for "scope and purpose" (92\%) and worse for "applicability" (57\%) were analyzed. Conclusion: The guidelines presented good overall quality, however, they need to improve their elaboration regarding applicability.
\end{abstract}

Keywords: postpartum period; Primary Health Care; clinical practice guideline.

Local de realização do estudo: Universidade Federal de Santa Catarina, Florianópolis/SC, Brasil.

Correspondência:Tatiane Baratieri. E-mail: baratieri.tatiane@gmail.com

Fonte de financiamento: nenhuma.

Conflito de interesses: nada a declarar.

Recebido em: Fev. 18, 2019. Aprovado em: Set. 06, 2020
Este é um artigo publicado em acesso aberto (Open Access) sob a licença Creative Commons Attribution, que permite uso, distribuição e reprodução em qualquer meio, sem restrições desde que o trabalho original seja corretamente citado. 


\section{INTRODUÇÃO}

As semanas após o nascimento do filho configuram-se como um período crítico para as mulheres, e necessitam de cuidados que irão impactar na saúde e bem-estar delas a longo prazo'. Durante esse período, as mulheres estão se adaptando a múltiplas mudanças, de ordens físicas, sociais e psicológicas, com recuperação do parto, ajuste hormonal, adaptação ao bebê e a seu papel de mãe ${ }^{2}$.

Os cuidados pós-parto devem se tornar um processo contínuo, em vez de um único encontro com serviços e apoio e, sobretudo, serem adaptados às necessidades individuais de cada mulher', e têm impactos positivos na morbimortalidade das mulheres nesse período 3 .

Os profissionais da Atenção Primária à Saúde (APS) são os principais responsáveis pela atenção às mulheres no período pós-parto, especialmente por esse ser o ponto de atenção mais próximo delas.

A fim de prestar um cuidado de qualidade e de acordo com as necessidades das mulheres, os profissionais precisam ter acesso e qualificação para o uso de diretrizes clínicas (guidelines), amparando assim suas ações de forma sistematizada. Tais guidelines, desenvolvidos em realidades distintas em diferentes países, instituições e organizações sobre essa temática, se constituem em um conjunto de recomendações baseadas em uma revisão sistemática da evidência e em avaliação dos riscos e benefícios das diferentes alternativas, com o objetivo de otimizar e qualificar a atenção à população $0^{5,6}$.

Para a elaboração de guidelines, deve-se utilizar as melhores evidências científicas disponíveis, considerar as preferências dos usuários e a experiência clínica de especialistas, verificar se são cientificamente válidos, utilizáveis e confiáveis, o que Ihes confere boa qualidade ${ }^{7,8}$.

Embora haja evidências crescentes de que guidelines podem melhorar os resultados clínicos e do sistema de saúde, seu grau de impacto na saúde das populações é influenciado pela qualidade e implementabilidade, pois seu conteúdo pode ser tendencioso, com recomendações pobres, não eficazes, potencialmente prejudiciais, ou difíceis de implementar ${ }^{9}$.

O reforço pela qualidade e rigor da rede de pesquisa em saúde é uma iniciativa internacional que visa melhorar a elaboração de guidelines. Recomendações atuais estão disponíveis para avaliar a qualidade e credibilidade das diretrizes clínicas, utilizando métodos para apoiar o desenvolvimento e avaliação confiável na elaboração dessas ${ }^{10}$.

Considerando o exposto, conhecer a qualidade dos guidelines para atenção pós-parto na APS é importante para aprimorar a atenção a essa população, impactando positivamente na saúde dela. Assim, o presente estudo tem por objetivo avaliar a qualidade de guidelines sobre a atenção às mulheres no pós-parto na APS.

\section{MÉTODO}

Trata-se de uma revisão sistemática da literatura, que foi conduzida e relatada de acordo com as diretrizes PRISMA (Principais Itens para Relatar Revisões Sistemáticas e Meta-análises). Com base na abordagem PICO, a estratégia da pesquisa considerou: população (mulher no pós-parto); intervenção (atenção à mulher no pós-parto na APS); comparação/controle (guidelines) e resultados (avaliação da qualidade das recomendações) ${ }^{11}$.

Os guidelines foram identificados por meio de busca na base de dados PubMed (Biblioteca Nacional de Medicina dos Estados Unidos), com os termos puerperium OR postnatal OR postpartum OR perinatal, limitado pelo termo "clinical guidelines"; e na base de dados Lilacs (Literatura Latino-Americana e do Caribe em Ciências da Saúde), com os descritores postpartum period OR período posparto OR período pós-parto AND clinical guideline OR guía de práctica clínica OR guia de prática clínica.

Considerando os poucos registros encontrados e tendo em vista que guidelines raramente são publicados em periódicos, foram incluídos outros bancos de dados eletrônicos como o National Guideline Clearinghouse (NGC), do National Institute for Health and Care Excellence (NICE), utilizando-se os mesmos termos de busca empregados na PubMed. Para incluir um 
maior número de evidências, optou-se por realizar busca na página do Google ${ }^{\circledR}$, utilizando-se os mesmos termos da Lilacs. A pesquisa ocorreu entre os meses de janeiro e fevereiro de 2018.

Foram elegíveis para o estudo os guidelines que atenderam aos seguintes critérios: tratar de recomendações sobre cuidados pós-parto de rotina e/ou complicações decorrentes do pósparto; contemplar em seu escopo responsabilidades da APS; ser publicado de 2008 a 2017, a fim de identificar recomendações com evidências mais atuais; estar disponível em texto completo; e ser publicado nos idiomas inglês, espanhol ou português. Foram excluídos guidelines quando encontrado o mesmo título publicado em edições/anos diferentes, eliminando-se o mais antigo bem como versões resumidas e guias rápidos, analisando-se somente a versão completa.

A busca e seleção dos estudos, assim como a avaliação dos guidelines foram realizadas por duas revisoras de forma independente, e as divergências foram revisadas e redefinidas a partir de consenso. Em todas as etapas, houve alto nível de concordância entre as revisoras.

Para a caracterização dos guidelines, foi elaborado um formulário para extração dos dados, digitados em uma planilha no Microsoft Excel ${ }^{\circledR}$ constando: ano, título, autor, instituição, país e objetivo. Posterior a isso, os guidelines foram submetidos a avaliação da qualidade.

A avaliação da qualidade baseou-se no instrumento Appraisal of Guidelines, Research, and Evaluation II (AGREE II) ${ }^{8}$, versão em português, composto por 23 itens, agrupados em seis domínios: 1) escopo e finalidade 2) envolvimento das partes interessadas (stakeholders), 3) rigor do desenvolvimento, 4) clareza e apresentação, 5) aplicabilidade e 6) independência editorial. Cada item foi avaliado em uma escala Likert de sete pontos em que 1 correspondia a discordo totalmente e 7 a concordo totalmente ${ }^{8}$. Para cada um dos seis domínios do AGREE II calculou-se uma pontuação de qualidade dividindo as diferenças entre a pontuação obtida e a pontuação mínima possível pela diferença entre as pontuações máxima e mínima possíveis, sendo que, quanto maior o escore, maior a qualidade ${ }^{8}$.

O domínio "Escopo e finalidade" diz respeito ao objetivo geral da diretriz, às questões específicas de saúde e à população-alvo; "Envolvimento das partes interessadas" focaliza em que medida a diretriz foi desenvolvida pelas partes interessadas adequadas e representa a visão dos usuários pretendidos; "Rigor do desenvolvimento" trata do processo usado para coletar e sintetizar as evidências, os métodos para a formulação das recomendações e a respectiva atualização dessas; "Clareza da apresentação" se refere a linguagem, estrutura e formato da diretriz; "Aplicabilidade" são os prováveis fatores facilitadores e barreiras para a implementação, estratégias para melhorar a aplicação, bem como envolvimento de recursos relacionados à utilização da diretriz; "Independência editorial" diz respeito à formulação das recomendações de modo a não terem vieses decorrentes de interesses conflitantes ${ }^{8}$.

A análise dos dados se deu por meio de estatística descritiva simples utilizando o programa Microsoft Excel ${ }^{\text {. }}$.

\section{RESULTADOS}

Um total de dez guidelines foram identificados para inclusão nessa revisão. A busca nas bases de dados forneceu um total de 689 estudos. Após os ajustes por duplicatas, 667 permaneceram. Desses, foram eliminados inicialmente 648, pois, nos "bancos de dados de buscas" não foram encontrados guidelines completos (314), e nas outras fontes os guidelines não estavam disponíveis (52), não tratavam sobre rotina de cuidados pós-parto (77), não contemplavam as responsabilidades da APS (163), ou foram publicados anteriormente ao ano de 2008 (83).

Dessa forma, 19 textos completos atenderam aos critérios de elegibilidade e foram examinados com mais detalhes. Cinco estudos foram excluídos por serem versões prévias, sendo mantidos os atualizados; quatro foram descartados por constituírem versões resumidas e guias rápidos, conforme observado na Figura 1.

Quanto à caracterização do material selecionado, de acordo com a Tabela 1, identificou-se que o ano de publicação variou de 2011 a 2017, sendo em sua maioria trabalhos publicados na Europa, com predomínio do Reino Unido. Quanto aos temas, apenas três guidelines ${ }^{5,12,13}$ tratam 


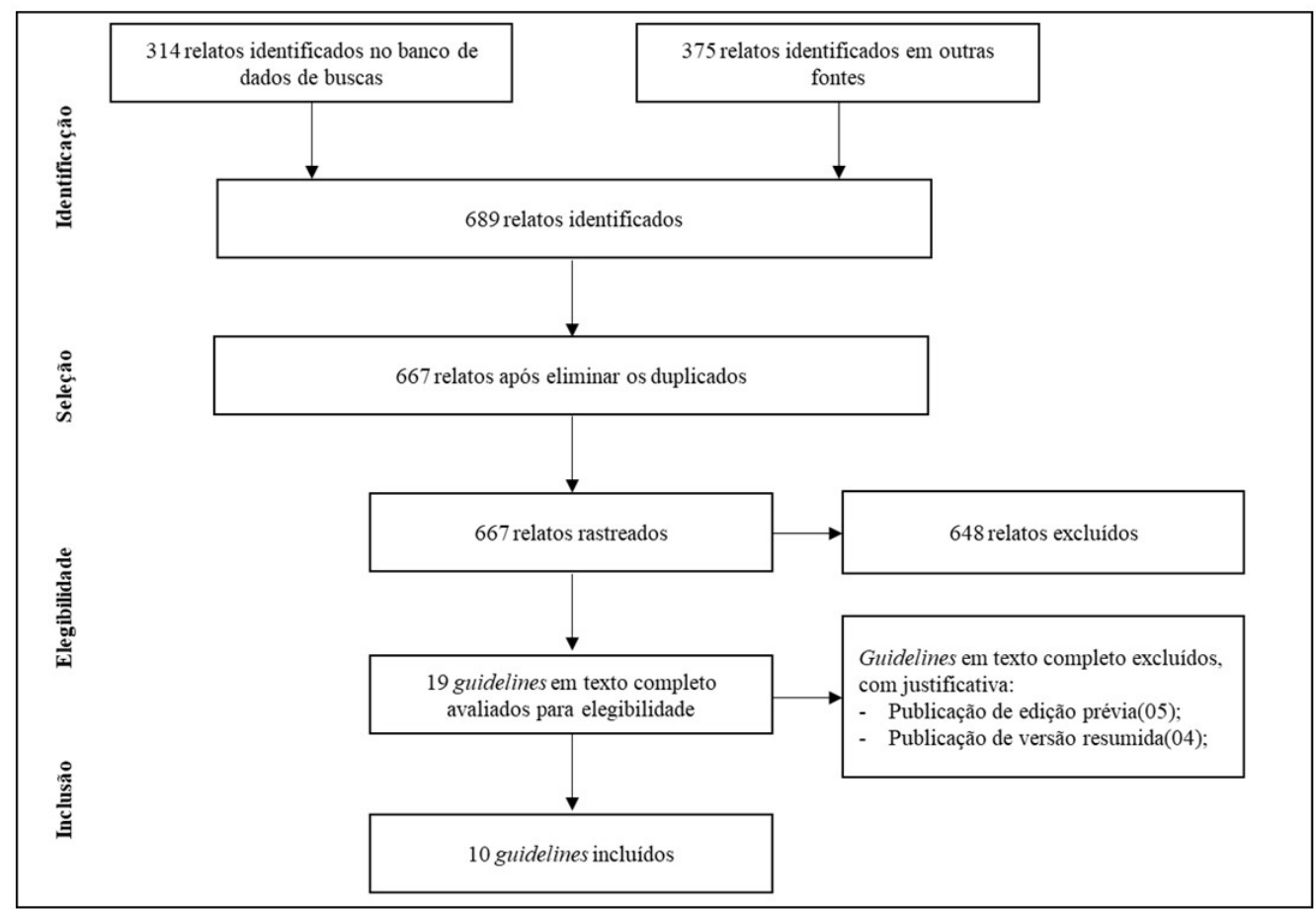

Figura 1. Fluxograma do processo de seleção dos guidelines

do cuidado geral às mulheres no puerpério, os demais referem-se a temas específicos, como saúde mental ${ }^{14-18}$, dor pélvica ${ }^{19}$ e contracepção ${ }^{20}$.

Ressalta-se que apenas o guideline da Organização Mundial da Saúde ${ }^{12}$ aborda especificamente ações para países de baixa renda, os demais são guidelines voltados para países desenvolvidos.

Quanto à avaliação da qualidade dos guidelines, de modo geral, foi boa (Tabela 2), com média de escore apresentando $77 \%$, de acordo com o instrumento AGREE II8.

Os domínios que receberam melhores escores foram "escopo e finalidade" $(92 \%)$, "rigor no desenvolvimento" (89\%) e "clareza e apresentação" (89\%). Em geral, os guidelines tinham uma proposta clara, com objetivo geral bem definido. Com exceção do $\mathrm{G}^{19}{ }^{19}$, os guidelines usaram métodos sistemáticos para revisão dos estudos, com critérios de seleção claramente descritos e consideraram os benefícios, efeitos colaterais e riscos para formular as recomendações, além de terem estabelecido uma relação coerente entre recomendações e evidências, e terem sido revisados por especialistas externos antes da publicação.

No item clareza da apresentação, o G4 ${ }^{12}$ teve como principal lacuna a falta de apresentação de ferramentas para aplicação, o $G 7^{13}$ não apresentou as recomendações-chave facilmente identificáveis e o G9 ${ }^{17}$ não apresentou de forma direta as diferentes opções para o gerenciamento da condição.

O domínio com pior resultado foi a "aplicabilidade", com pontuação moderada (57\%) na média geral. A principal fragilidade nesse domínio é a falta de apresentação do critério de revisão para monitoramento e/ou auditoria do guideline, sendo que somente dois ${ }^{15,20}$ cumpriram

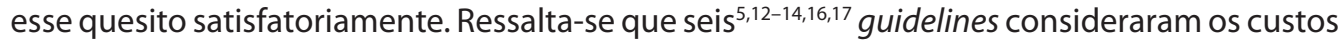
para a aplicação das recomendações, e três ${ }^{13,15,19}$ não discutiram barreiras organizacionais na aplicação das recomendações.

No domínio "independência editorial", três $s^{5,12,15}$ não relataram sobre o guideline ser editorialmente independente quanto ao financiamento, e um ${ }^{12}$ não descreveu sobre conflitos de interesse. Um guideline $e^{19}$ obteve pontuação zero nesse domínio. 
Tabela 1. Caracterização dos guidelines selecionados. Florianópolis/SC, 2018

\begin{tabular}{|c|c|c|c|}
\hline Ident. & Título/Instituição & País/Ano & Objetivo \\
\hline $\mathrm{G} 1^{14}$ & $\begin{array}{l}\text { Depression and related disorders - anxiety, } \\
\text { bipolar disorder and puerperal psychosis - in the } \\
\text { perinatal period / Beyond blue }\end{array}$ & Austrália 2011 & $\begin{array}{l}\text { Fornecer recomendações que apoiem a } \\
\text { identificação precoce e o gerenciamento efetivo } \\
\text { de problemas de saúde mental no período } \\
\text { perinatal, além de objetivar melhores resultados } \\
\text { de saúde mental para as mulheres e suas famílias. }\end{array}$ \\
\hline $\mathrm{G} 2^{18}$ & $\begin{array}{l}\text { Guía de Práctica Clínica para el tratamento de la } \\
\text { depresión em Atención Primaria }\end{array}$ & Espanha 2011 & $\begin{array}{l}\text { Ajudar na tomada de decisões para o tratamento } \\
\text { de pacientes adultos com depressão no ambiente } \\
\text { de cuidados primários. }\end{array}$ \\
\hline $\mathrm{G}^{15}$ & $\begin{array}{l}\text { Management of perinatal mood disorders: } \\
\text { A national clinical guideline / Scottish } \\
\text { Intercollegiate Guidelines Network }\end{array}$ & $\begin{array}{l}\text { Reino Unido } \\
2012\end{array}$ & $\begin{array}{l}\text { Fornecer recomendações sobre Depressão Pós } \\
\text { Parto (DPP), abrangendo a previsão, detecção } \\
\text { e prevenção, bem como o gerenciamento nos } \\
\text { cuidados primários e secundários. }\end{array}$ \\
\hline $\mathrm{G}^{12}$ & $\begin{array}{l}\text { WHO recommendations on postnatal care of the } \\
\text { mother and newborn / World Health Organization } \\
\text { (WHO) }\end{array}$ & Suíça 2013 & $\begin{array}{l}\text { Fornecer recomendações para cuidados pós- } \\
\text { natal de mães e recém-nascidos para melhorar a } \\
\text { qualidade dos cuidados pós-parto e resultados de } \\
\text { saúde para mães e recém-nascidos. }\end{array}$ \\
\hline $\mathrm{G} 5^{19}$ & $\begin{array}{l}\text { Management of pelvic girdle pain in pregnancy } \\
\text { and post-partum / Institute of Obstetrician \& } \\
\text { Gynaecologists / Royal College Physicians of } \\
\text { Ireland }\end{array}$ & Irlanda 2014 & $\begin{array}{l}\text { Auxiliar os profissionais de saúde no diagnóstico } \\
\text { de dor de cintura pélvica (DCP) com opções de } \\
\text { manejo da gravidez, trabalho, parto, pós-natal. }\end{array}$ \\
\hline $\mathrm{G} 6^{5}$ & $\begin{array}{l}\text { Guía de práctica clínica de atención em el } \\
\text { embarazo y puerperio }\end{array}$ & Espanha 2014 & $\begin{array}{l}\text { Estabelecer um conjunto de recomendações } \\
\text { baseadas na evidência científica de mais alta } \\
\text { qualidade disponível para melhorar a saúde das } \\
\text { mulheres grávidas e puérperas, bem como a } \\
\text { saúde dos recém-nascidos. }\end{array}$ \\
\hline $\mathrm{G} 7^{13}$ & $\begin{array}{l}\text { Postnatal care up to } 8 \text { weeks after birth / National } \\
\text { Institute for Health and Care Excellence }\end{array}$ & $\begin{array}{l}\text { Reino Unido } \\
2015\end{array}$ & $\begin{array}{l}\text { Identificar o cuidado de rotina que toda mulher } \\
\text { e seu bebê devem receber nas primeiras 6-8 } \\
\text { semanas após o nascimento, com base nas } \\
\text { melhores evidências disponíveis, além de abordar } \\
\text { as recomendações sobre o co-sleeping e síndrome } \\
\text { e morte súbita do lactente até o primeiro ano da } \\
\text { vida da criança. }\end{array}$ \\
\hline $\mathrm{G}^{16}$ & $\begin{array}{l}\text { Antenatal and postnatal mental health: clinical } \\
\text { management and service guidance / National } \\
\text { Institute for Health and Care Excellence }\end{array}$ & $\begin{array}{l}\text { Reino Unido } \\
2015\end{array}$ & $\begin{array}{l}\text { Recomendar o reconhecimento, avaliação, } \\
\text { cuidados e tratamento de problemas de saúde } \\
\text { mental em mulheres durante a gravidez e o } \\
\text { período pós-natal (até um ano após o parto) e em } \\
\text { mulheres que estão planejando engravidar. }\end{array}$ \\
\hline G9 ${ }^{17}$ & $\begin{array}{l}\text { Adult depression in primary care / Institute for } \\
\text { Clinical Systems Improvement (ICSI }\end{array}$ & $\begin{array}{l}\text { Estados } \\
\text { Unidos } 2016\end{array}$ & $\begin{array}{l}\text { Apresentar as recomendações de cuidado na } \\
\text { atenção primária para diagnóstico e tratamento } \\
\text { da depressão em adultos com idade igual ou } \\
\text { superior a } 18 \text { anos. }\end{array}$ \\
\hline $\mathrm{G} 10^{20}$ & $\begin{array}{l}\text { FSRH Guideline: Contraception after pregnancy / } \\
\text { Faculty of Sexual and Reproductive Health (FSRH) }\end{array}$ & $\begin{array}{l}\text { Inglaterra } \\
2017\end{array}$ & $\begin{array}{l}\text { Fornecer recomendações para o uso seguro de } \\
\text { contracepção, incluindo categorias para mulheres } \\
\text { após o parto (pós-parto), aborto (incluindo } \\
\text { aborto espontâneo), mulheres com história de } \\
\text { gravidez ectópica ou com doença tromboblástica } \\
\text { gestacional. }\end{array}$ \\
\hline
\end{tabular}

Em relação ao "envolvimento dos interessados", nenhum guideline relatou sobre a realização de teste piloto entre os usuários do material, e três ${ }^{12,18,19}$ não buscaram a visão e as preferências dos pacientes para elaboração do guideline.

\section{DISCUSSÃO}

Os guidelines apresentaram boa qualidade geral, com destaque para os domínios "escopo e finalidade", "rigor no desenvolvimento" e"clareza e apresentação". Entretanto, o pior domínio avaliado foi a "aplicabilidade" que se refere à discussão das mudanças organizacionais e as implicações de custos de aplicar as recomendações e apresentação de critérios de revisão para monitorar o uso das diretrizes ${ }^{7}$, corroborando com outros estudos de avaliação de guidelines ${ }^{22-24}$.

A baixa aplicabilidade dos guidelines é um fator preocupante, pois constitui um quesito essencial para que as recomendações sejam incorporadas à prática dos profissionais da APS. Guidelines elaborados de acordo com a realidade em cada contexto da APS fomentam a tomada 
Tabela 2. Escores por domínios e avaliação geral dos guidelines (\%). Florianópolis/SC, 2018

\begin{tabular}{|c|c|c|c|c|c|c|c|c|c|c|c|}
\hline Domínios & $\mathbf{G} 1^{14}$ & $\mathbf{G} 2^{18}$ & $\mathbf{G 3}^{15}$ & G4 ${ }^{12}$ & $\mathbf{G 5}^{19}$ & $\mathbf{G 6}^{5}$ & G7 ${ }^{13 *}$ & G8 ${ }^{16 *}$ & G917 & G10 20 & Média \\
\hline Escopo e finalidade & 100 & 100 & 78 & 89 & 89 & 89 & 100 & 100 & 89 & 89 & 92 \\
\hline Envolvimento dos interessados & 75 & 50 & 75 & 58 & 33 & 75 & 75 & 75 & 75 & 75 & 67 \\
\hline Rigor no desenvolvimento & 100 & 90 & 86 & 100 & 38 & 100 & 90 & 100 & 90 & 100 & 89 \\
\hline Clareza e apresentação & 100 & 100 & 92 & 58 & 100 & 100 & 75 & 92 & 75 & 100 & 89 \\
\hline Aplicabilidade & 67 & 67 & 44 & 78 & 11 & 78 & 44 & 56 & 67 & 56 & 57 \\
\hline Independência editorial & 100 & 67 & 33 & 17 & - & 67 & 83 & 83 & 100 & 100 & 65 \\
\hline Avaliação geral & 90 & 79 & 68 & 67 & 45 & 85 & 78 & 84 & 83 & 87 & 77 \\
\hline
\end{tabular}

*G7 e G8 por se tratar de versões atualizadas, contemplando somente as recomendações e não o guideline completo, sua avaliação foi complementada por suas versões completas $\mathrm{NICE}^{21,4}$, respectivamente

de decisões efetivas e seguras, otimizam a assistência e auxiliam na prestação da atenção integral às mulheres no pós-parto, o que confere melhor qualidade ao cuidado ${ }^{5,13,25}$.

Assim, não basta elaborar diretrizes com qualidade metodológica, pois, se estas não forem clinicamente aplicáveis, implicam na não implementação no contexto assistencial ${ }^{22}$, visto que precisam atingir o público-alvo que está inserido na realidade, com custo viável para implantação.

No Brasil e internacionalmente, em geral, a assistência pós-parto é negligenciada, com diretrizes e protocolos que se restringem ao puerpério imediato e tardio, não abordam as mulheres no período remoto na APS e focam na atenção materno-infantil e reprodutiva ${ }^{26}, 0$ que dificulta sua aplicabilidade no que tange a assistência integral.

Outro aspecto a ser melhorado é a necessidade de ampliar o envolvimento dos potenciais usuários. A efetivação desse domínio favorece o melhor desenvolvimento da aplicabilidade do guideline, já que a participação dos potenciais usuários no material é fundamental para elaborar recomendações pautadas na realidade das mulheres e fomentar o uso delas. Cabe destacar o $6^{5}$ que obteve pontuação máxima nesse quesito avaliativo, por considerar a visão e preferências dos usuários e, para tal, é orientado por um manual metodológico ${ }^{27}$ que aborda as implicações dos envolvidos no desenvolvimento de guidelines.

Estudos nacionais e internacionais apontam para a invisibilidade das mulheres no período pós-parto na APS, visto que, após o nascimento, as mulheres deixam de ser priorizadas, sendo a assistência direcionada especialmente para a criança ${ }^{2,28}$. Ressalta-se que o fortalecimento da APS se dá por meio da participação dos usuários ${ }^{25}$. O envolvimento das mulheres no âmbito da APS, a fim de conhecer suas necessidades de saúde, é um aspecto fundamental para melhorar a atenção pós-parto.

Os dados analisados no presente estudo refletem a necessidade de melhorar a qualidade das recomendações pautadas em instrumentos conhecidos e validados cientificamente. $O$ instrumento AGREE $\|^{8}$ pode auxiliar comitês organizadores de guidelines e viabilizar uma estrutura para revisar a qualidade de guidelines, bem como o presente estudo, fornecendo suporte para melhorar a qualidade das recomendações realizadas. Nesta revisão, apenas quatro guidelines ${ }^{5,13,16,18}$ deixaram explícito que utilizaram o AGREE para avaliar a qualidade do material, e um ${ }^{17}$ seguiu um protocolo baseado em critérios semelhantes ao AGREE, no entanto, não o citou.

O estudo também evidenciou que a escassez de guidelines abrangentes para mulheres no pós-parto é uma preocupação, devido ao estresse que muitas vivenciam nesse período, atrelado ao alto fardo da morbidade materna pós-parto ${ }^{2,29,30}$.

O desenvolvimento de guidelines abrangentes e de alta qualidade é uma atividade dispendiosa em tempo e recursos, e isso pode ser uma das razões para a escassez de guidelines nessa área ${ }^{6,30}$, especialmente em países em desenvolvimento. Esses, em geral, têm poucos recursos disponibilizados para ciência e tecnologia, e acabam recorrendo a guidelines internacionais para embasar sua prática ${ }^{6}$. 
No Brasil há um protocolo nacional de atenção integral à saúde das mulheres ${ }^{31}$ que aborda a atenção pós-parto na APS, porém, ele não segue o rigor metodológico de um guideline, o que pode limitar seu uso na prática assistencial pelos profissionais da APS.

Este estudo apresentou algumas limitações no seu desenvolvimento. A primeira foi a exclusão de diretrizes que não estavam disponíveis para download pela internet, o que aconteceu especialmente para países da América Latina. No entanto, guidelines são elaborados para orientar a prática clínica e devem estar prontamente disponíveis para aqueles que precisam utilizá-las. A segunda limitação é a inclusão de guidelines somente em português, inglês e espanhol, que, apesar de serem idiomas que abrangem grande parcela da população mundial, podem ter excluído realidades importantes da análise. O terceiro aspecto refere-se à ferramenta AGREE $\|^{8}$ que tem vários pontos da escala de Likert que podem aludir a um sistema de avaliação subjetiva. Apesar disso, o manual do AGREE $\|^{8}$ é robusto em suas instruções sobre a marcação de cada componente nos vários domínios e a concordância entre os dois avaliadores deste estudo foi alta, o que sugere a validade do instrumento e do seu uso.

Os guidelines analisados receberam boa avaliação geral, sendo mais bem avaliados no domínio "escopo e finalidade" e pior avaliados no domínio "aplicabilidade". A maior parte dos guidelines trata de temas específicos, não abordando integralmente as mulheres no pós-parto, e apenas um foi direcionado para países em desenvolvimento.

Considerando a avaliação dos guidelines, este estudo traz como principais recomendações: melhorar os quesitos quanto à aplicabilidade dos guidelines, especialmente com implementação de estudos piloto e auditoria para monitorar a aceitação e feedback quanto ao uso dos guidelines; implementar guidelines que abordem de maneira integral as mulheres no pós-parto na APS e; incentivar a elaboração de guidelines em países em desenvolvimento.

Existe uma demanda significativa para melhorias no desenvolvimento de diretrizes de alta qualidade, o que justifica urgentemente a implementação de pesquisas que minimizem as lacunas vitais nas evidências para a formulação de guidelines para atender às mulheres no pós-parto no âmbito da APS.

\section{REFERÊNCIAS}

1. McKinney J, Keyser L, Clinton S, Pagliano C. ACOG Committee Opinion No. 736: Optimizing postpartum care. Obstetrics \& Gynecology. 2018;132(3):784-785.

2. Corrêa MSM, Feliciano KVO, Pedrosa EN, Souza Al. Acolhimento no cuidado à saúde da mulher no puerpério. Cad Saude Publica. 2017;33(3):e00136215. http://dx.doi.org/10.1590/0102-311×00136215. PMid:28380144.

3. Langlois ÉV, Miszkurka M, Zunzunegui MV, Ghaffar A, Ziegler D, Karp I. Inequities in postnatal care in low-and middle-income countries: a systematic review and meta-analysis. Bull World Health Organ. 2015;93(4):259-70. http://dx.doi.org/10.2471/BLT.14.140996.

4. National Institute for Health and Care Excellence. Antenatal and postnatal mental health: clinical management and service guidance. Leicester: The British Psychological Societyand The Royal College of Psychiatrists; 2014.

5. Espanha. Ministerio de Sanidad, Servicios Sociales e Igualdad, Agencia de Evaluación de Tecnologías Sanitarias de Andalucía. Guía de práctica clínica de atención em el embarazo y puerperio. Guías de Práctica Clínica en el SNS: AETSA 2011/10. Andalucía: Ministerio de Sanidad, Servicios Sociales e Igualdad; 2014.

6. Mehndiratta A, Sharma S, Gupta N, Sankar M, Cluzeau F. Adapting clinical guidelines in India_-a pragmatic approach. BMJ. 2017;359:j5147. http://dx.doi.org/10.1136/bmj.j5147. PMid:29150419.

7. AGREE Collaboration. development and validation of an international appraisal instrument for assessing the quality of clinical practice guidelines: the AGREE project. Qual Saf Health Care. 2003;12(1):18-23. http:// dx.doi.org/10.1136/qhc.12.1.18. PMid:12571340.

8. Appraisal of Guidelines for Research and Evaluation. The AGREE II Instrument [Internet]. 2009 [citado em 2019 Fev 18]. Disponível em: http://www.agreetrust.org

9. Chen Y, Yang K, Marušić A, Qaseem A, Meerpohl JJ, Flottorp S, et al. A reporting tool for practice guidelines in health care: the RIGHT statement. Ann Intern Med. 2017;166(2):128-32. http://dx.doi.org/10.7326/ M16-1565. PMid:27893062.

10. Brouwers MC, Florez ID, McNair SA, Vella ET, Yao X. Clinical Practice Guidelines: tools to support high quality patient care. Semin Nucl Med. 2018;49(2):145-152. PMid:30819394. 
11. Moher D, Liberati A, Tetzlaff J, Altman DG. Principais itens para relatar revisões sistemáticas e metaanálises: a recomendação PRISMA. Epidemiol Serv Saude. 2015;24(2):335-42. http://dx.doi.org/10.5123/ S1679-49742015000200017.

12. World Health Organization. WHO recommendations on Postnatal care of the mother and newborn. Geneva:WHO; 2013.

13. National Institute for Health and Care Excellence. Postnatal care up to 8 weeks after birth [Internet] London: NICE; 2015 [citado 2019 Fev 9]. Disponível em: https://www.nice.org.uk/guidance/cg37

14. Beyondblue, organizador. Clinical practice guidelines for depression and related disorders - anxiety, bipolar disorder and puerperal psychosis - in the perinatal period. A guideline for primary care health professionals. Melbourn: Beyondblue; 2011.

15. Scottish Intercollegiate Guidelines Network. Management of perinatal mood disorders [Internet]. SIGN; 2012 [citado 2019 Fev 9]. Disponível em: http://www.sign.ac.uk

16. National Institute for Health and Care Excellence. Antenatal and postnatal mental health: clinical management and service guidance [Internet]. Leicester: British Psychological Society; 2015 [citado 2019 Fev 9]. Disponível em: https://www.nice.org.uk/guidance/cg192

17. Trangle M, Gursky J, Haight R, Hardwig J, Hinnenkamp T, Kessler D, et al. Adult depression in primary care. Bloomington: Institute for Clinical Systems Improvement (ICSI); 2016.

18. García-Herrera PB, Nogueras ME V, Muñoz CF, Morales AJM. Guía de Práctica Clínica para el tratamento de la depresión em Atención Primaria. Málaga: UGC Salud Mental; 2011.

19. Institute of Obstetrician \& Gynaecologists. Royal College Physicians of Irland. Management of pelvic girdle pain in pregnacy and postpartum [Internet]. Irland: Institute of Obstetrician \& Gynaecologists; 2014 [citado 2019 Fev 9]. Disponível em: https://www.hse.ie/eng/services/publications/clinical-strategy-andprogrammes/management-of-pelvic-girdle-pain-in-pregnancy-and-post-partum.pdf

20. Faculty of Sexual and Reproductive Health. Contraception after pregnancy [Internet]. London: FSRH; 2017 [citado 2019 Fev 9]. Disponível em: https://www.fsrh.org/news/new-fsrh-guideline--contraception-afterpregnancy

21. Demott K, Bick D, Norman R, Ritchie G, Turnbull N, Adams C, et al. Clinical guidelines and evidence review for post natal care: routine post natal care of recently delivered women and their babies. London: NICE; 2006.

22. Sabharwal S, Patel NK, Gauher S, Holloway I, Athanasiou T. High methodologic quality but poor applicability: Assessment of the AAOS guidelines using the AGREE II instrument. Clin Orthop Relat Res. 2014;472(6):1982-8. http://dx.doi.org/10.1007/s11999-014-3530-0. PMid:24566890.

23. Anaya MVM, Franco JV, Merchán-Galvis ÁM, Gallardo CR, Cosp XB. Quality assessment of clinical practice guidelines on treatments for oral cancer. Cancer Treat Rev. 2018;65(29):47-53. http://dx.doi.org/10.1016/j. ctrv.2018.03.001. PMid:29547765.

24. Wu D, Jiang W, Yu L, Wang Y, Tao Y, Tang H, et al. Quality assessment of clinical practice guidelines for infectious diseases in China. J Evid Based Med. 2018;11(2):95-100. http://dx.doi.org/10.1111/jebm.12293. PMid:29464853.

25. Tasca R, Massuda A, Carvalho WM, Buchweitz C, Harzheim E. Recomendações para o fortalecimento da atenção primária à saúde no Brasil. Rev Panam Salud Publica. 2020;44:e4. http://dx.doi.org/10.26633/ RPSP.2020.4

26. Baratieri T, Natal S. Postpartum program actions in primary health care: an integrative review. Cien Saude Colet. 2019;24(11):4227-38. http://dx.doi.org/10.1590/1413-812320182411.28112017. PMid:31664395.

27. Espanha. Grupo de trabajo de implicación de pacientes em el desarrollo de GPC. Implicación de pacientes em el desarrollo de guías de práctica clínica: manual metodológico. Guías de Práctica Clínica en el SNS: IACS No 2010/01 [Internet]. Andalucía: Ministerio de Sanidad, Servicios Sociales e lgualdad; 2011 [citado 2019 Fev 9]. Disponível em: http://portal.guiasalud.es/web/guest/guias-practica-clinica

28. Justino GBS, Soares GCF, Baraldi GN, Teixeira MCI, Salim RN. Sexual and reproductive health in the puerperium: women's experiences. J Nurs UFPE. 2019;13:e240054.

29. Souza $A B Q$, Fernandes MB. Diretriz para assistência de enfermagem: ferramenta eficaz para a promoção da saúde no puerpério. Rev RENE. 2014;15(4):594-604.

30. Haran C, Van Driel M, Mitchell BL, Brodribb WE. Clinical guidelines for postpartum women and infants in primary care-a systematic review. BMC Pregnancy Childbirth. 2014;14:51. http://dx.doi.org/10.1186/14712393-14-51. PMid:24475888.

31. Brasil. Ministério da Saúde. Protocolos da Atenção Básica: saúde das mulheres. Brasília: Ministério da Saúde; 2016 